\title{
Modeling the Uniformity of Manifold with Various Configurations
}

\author{
Jafar M. Hassan, ${ }^{1}$ Thamer A. Mohamed, ${ }^{2}$ Wahid S. Mohammed, ${ }^{1}$ and Wissam H. Alawee ${ }^{1}$ \\ ${ }^{1}$ Department of Mechanical Engineering, University of Technology, Baghdad, Iraq \\ ${ }^{2}$ Department of Civil Engineering, Faculty of Engineering, Universiti Putra Malaysia (UPM), 43400 Serdang, Selangor, Malaysia
}

Correspondence should be addressed to Jafar M. Hassan; jafarmehdi1951@yahoo.com

Received 14 February 2014; Accepted 24 July 2014; Published 24 August 2014

Academic Editor: Mohy S. Mansour

Copyright (C) 2014 Jafar M. Hassan et al. This is an open access article distributed under the Creative Commons Attribution License, which permits unrestricted use, distribution, and reproduction in any medium, provided the original work is properly cited.

\begin{abstract}
The flow distribution in manifolds is highly dependent on inlet pressure, configuration, and total inlet flow to the manifold. The flow from a manifold has many applications and in various fields of engineering such as civil, mechanical, and chemical engineering. In this study, physical and numerical models were employed to study the uniformity of the flow distribution from manifold with various configurations. The physical model consists of main manifold with uniform longitudinal section having diameter of $10.16 \mathrm{~cm}$ (4 in), five laterals with diameter of $5.08 \mathrm{~cm}$ ( 2 in), and spacing of $22 \mathrm{~cm}$. Different inlet flows were tested and the values of these flows are 500, 750, and $1000 \mathrm{~L} / \mathrm{min}$. A manifold with tapered longitudinal section having inlet diameters of $10.16 \mathrm{~cm}(4 \mathrm{in})$ and dead end diameter of $5.08 \mathrm{~cm}(2 \mathrm{in})$ with the same above later specifications and flow rates was tested for its uniformity too. The percentage of absolute mean deviation for manifold with uniform diameter was found to be $34 \%$ while its value for the manifold with nonuniform diameter was found to be $14 \%$. This result confirms the efficiency of the nonuniform distribution of fluids.
\end{abstract}

\section{Introduction}

Flow in manifold is of great importance in many industrial processes when it is necessary to distribute a large fluid stream into several smaller streams and then to collect them into one discharge stream. Manifolds can usually be categorized into one of the following types [1]: dividing, combining, parallel, and reverse flow manifolds as shown in Figure 1. Parallel and reverse flow manifolds are those which combine dividing and combining flow manifolds and are most commonly used in plate heat exchangers. In a parallel flow manifold, the flow directions in dividing and combining flow headers are the same which is generally referred to as a $Z$-manifold. In a reverse flow manifold, the flow directions are opposite and it is referred to as a $U$-manifold. A uniform flow distribution requirement is a common issue in many engineering circumstances such as plate-type heat exchangers, piping system, heat sinks for cooling of electronic devices, fuel cells, chemical reactors, solar thermal collectors, flow distribution systems in treatment plant, and the piping system of pumping stations. Therefore, for most applications, the goal of manifold design is to achieve a uniform flow distribution through all of the lateral exit ports. A great number of experimental, analytical, and numerical studies deal with flow in manifold.

The flow in distribution manifold has been studied by several investigators [2-6]. For instance, Bajura [2] developed the general theoretical model for investigation of the performance of single-phase flow distribution for both intake and exhaust manifolds. Bajura and Jones Jr. [3] extended the previous model and the prediction for the flow rates and the pressures in the headers of dividing, combining, reverse, and parallel manifold configurations. Majumdar [4] developed a mathematical model with one-dimensional elliptic solution procedure for predicting flows in dividing and combining flow manifolds. Bassiouny and Martin [5, 6] presented an analytical solution for the prediction of flow and pressure distribution in both intake and exhaust conduits of heat exchanger for both types flow ( $U$-type and $Z$-type). A great number of experimental and numerical studies covered the effect of design parameters on flow distribution in manifold. Choi et al. $[7,8]$ studied numerically the effect of Reynolds number and the width ratio on the flow distribution in manifolds of a liquid cooling module for 
electronic packaging. Kim et al. [9] investigated numerically the effects of the header shapes and Reynolds number on the flow distribution in a parallel flow manifold of a liquid cooling module for electronic packaging, for three different header geometries (i.e., rectangular, triangular, and trapezoidal) with the $Z$-type flow direction. Jiao et al. [10] investigated experimentally the effect of the inlet pipe diameter, the first header's diameter of equivalent area, and the second header's diameter of equivalent area on the flow maldistribution in plate-fin heat exchanger. Wen et al. [11] investigated flow characteristics in the entrance region of plate-fin heat exchanger by means of particle image velocimetry (PIV). Tong et al. [12] investigated numerically the strategies capable of perfecting manifold design to achieve the same rate of mass outflow through each of the exit ports of a distribution manifold. Minqiang et al. [13] performed a three-dimensional computational fluid dynamics (CFD) model to calculate the velocity distribution among multiple parallel microchannels with triangle manifolds. The effect of channel width and channel spacing on flow distribution among microchannels with U-shape rectangular manifolds has been investigated by Mathew et al. [14]. Chen and Sparrow [15] present a method to investigate the effect of geometric shape of the exit ports on mass flow rate uniformity effusing from a distribution manifold; three candidate exitport geometries were considered: (a) an array of discrete slots, (b) an array of discrete circular apertures, and (c) a single continuous longitudinal rectangular slot. In order to have a valid comparison of the impacts of these individual geometries, the total exit areas were made identical. Dharaiya et al. [16] studied numerically the effect of tapered header configuration to reduce flow maldistribution in minichannels and microchannels. Tong et al. [17] applied a logic-based systematic method of designing manifold systems to achieve flow rate uniformity among the channels that interconnect a distribution manifold and a collection manifold. The method was based on tailoring the flow resistance of the individual channels to achieve equal pressure drops for all the channels. The tailoring of the flow resistance was accomplished by the use of gate-valve-like obstructions. Huang and Wang [18] examined an inverse design problem to determine the optimum variables for a three-dimensional $Z$-type compact parallel flow heat exchanger with the Levenberg-Marquardt method (LMM) [19]. To obtain the uniform tube flow rates, five different optimization design problems were examined to demonstrate the validity of the study. Wang et al. [20] investigated experimentally and numerically the single-phase flow into parallel flow heat exchangers with inlet and outlet rectangular headers having square cross-section and 9 circular tubes. Wang et al. [21] presented experimentally the results of liquid flow distribution in compact parallel flow heat exchanger through a rectangular and 5 modified inlet headers (i.e., 1 trapezoidal, one multistep, 2 baffle plates, and 1 baffle tubes header). Zeng et al. [22] performed a three-dimensional computational fluid dynamics (CFD) model to calculate the velocity distribution among microchannels with two different manifold structures. A similar performance improvement with a more uniform flow distribution in methanol steam reformers was reported by Jang et al. [23]. Such findings affirm the influence of flow distribution uniformity on

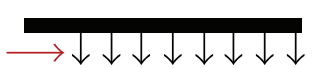

(a)

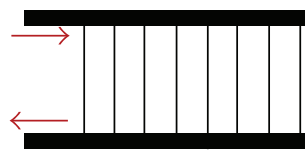

(c)

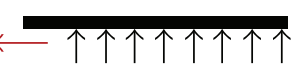

(b)

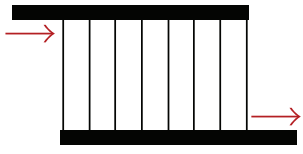

(d)
FIGURE 1: Different types of flow manifolds: (a) dividing, (b) combining, (c) $U$-manifold, and (d) $Z$-manifold.

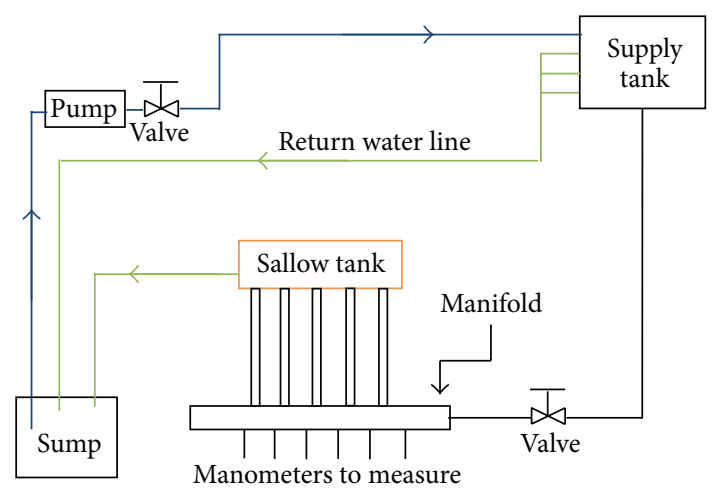

FIGURE 2: Experimental setup.

the performance of microreactor devices and reflect the importance of efficient manifold design. Tuo and Hrnjak [24] investigated experimentally and numerically the flow maldistribution caused by the pressure drop in headers and its impact on the performance of a microchannel evaporator with horizontal headers and vertically oriented tubes. Kim and Byun [25] studied experimentally the effect of inlet configuration on upward branching of two-phase refrigerant in a parallel flow heat exchanger. Three different inlet orientations (parallel, normal, and vertical) were investigated.

In general, all previous studies for manifolds with different applications had shown that typical manifold design does not give a uniform flow distribution at outlets. Therefore, the objective of the study was to predict the flow distribution through each outlet for circular cross-section header and to develop an optimized tapered cross-section header design having a better flow distribution through outlets.

\section{Methodology}

2.1. Experimental Setup. The nonuniformity of flow distribution through parallel outlets is found to be more severe in models with constant cross-sectional area headers [16]. Hence, the objectives of the study are to predict the flow distribution through each outlet of manifold with uniform longitudinal section and to develop an optimized manifold with longitudinal section design having a better flow distribution through outlets. The schematic diagram of the experimental setup is shown in Figure 2. The experimental setup consists of water tank with over flow, steel support, 


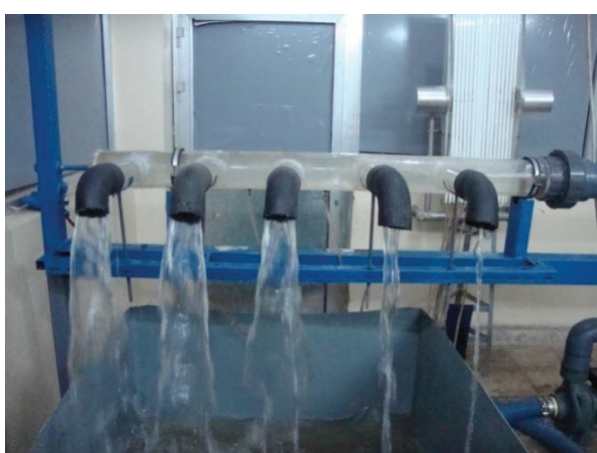

(a)

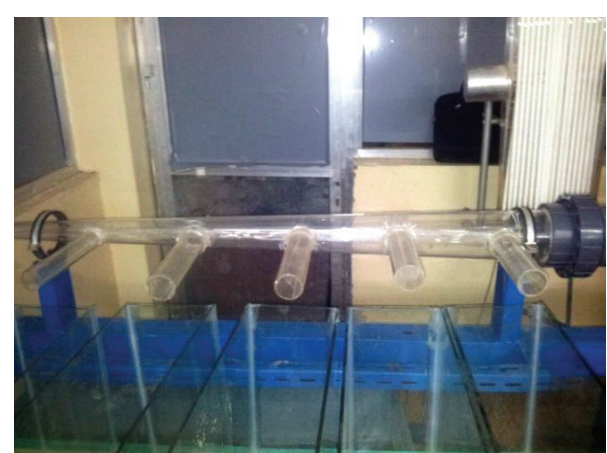

(b)

FIGURE 3: Experimental setup of two manifold configurations: (a) manifold with uniform longitudinal section and (b) manifold with tapered longitudinal section.

pump, sump, and number of valves to set the required flow rate through two dividing manifolds. The first manifold is with uniform longitudinal section while the second manifold is with tapered longitudinal section (optimal taper shape from numerical section).

The rig was assembled at a selected site in fluid laboratory of Machines and Equipment Engineering Department, University of Technology, Iraq. The water tank is rested on $3 \mathrm{~m}$ high steel elevated frame. At the outlet of each branch pipe, a shallow tank with cross-section $150 \mathrm{~cm} \times 150 \mathrm{~cm}$ is used to collect the water flowing from the branch pipes as shown in Figure 3. The water from the branch pipes is measured using 50 liter capacity rectangular tank. A constant head was ensured during the experiments and, as a result, constant flow rate from branch pipes was obtained. Six uniformly spaced piezometers were installed along the pipe to monitor the pressure head at the branch pipes. The spacing was $25 \mathrm{~cm}$.

Dimensions of two configuration manifolds are shown in Figure 4. The manifolds have been fabricated with acrylic material to ensure the developed flow and the good visibility of flow pattern. The branch pipes junctions are at right angles with header. The difference between two models only lies in the header configuration.

2.2. CFD Model. In the CFD analysis, a model of the manifold with uniform longitudinal section was prepared. The configuration used in the analysis is as shown in Figure 1(a). Later, the simulation was performed to develop a manifold design to achieve nearly uniform flow distribution through the outlets. The geometry of manifold with tapered longitudinal section is shown in Figure 1(b). The manifold diameter ratio $\left(D_{1} / D_{2}\right)$ is varied parametrically to estimate the optimal tapered ratio and uniform flow distribution.

In the present problem, the fluid flow is threedimensional; that is, all three possible velocity components $(x, y$, and $z)$ exist and all three components depend on the three coordinates of cartesian geometry. The statements of the governing parietal equations are

$$
\frac{\partial u}{\partial x}+\frac{\partial v}{\partial y}+\frac{\partial w}{\partial z}=0 .
$$

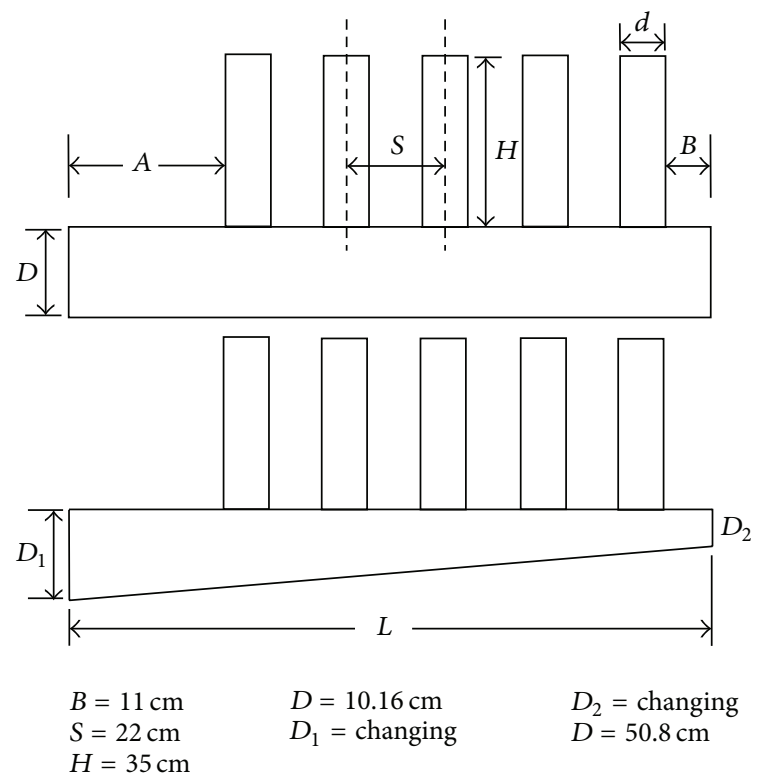

FIgURE 4: Manifolds used for conducting experiment.

$x$-momentum is

$$
\begin{aligned}
P & {\left[\frac{\partial}{\partial x}\left(u^{2}\right)+\frac{\partial}{\partial y}(u v)+\frac{\partial}{\partial z}(u w)\right] } \\
= & -\frac{\partial p}{\partial x}+\frac{\partial}{\partial x}\left(\mu_{\mathrm{eff}} \frac{\partial u}{\partial x}\right)+\frac{\partial}{\partial y}\left(\mu_{\mathrm{eff}} \frac{\partial u}{\partial y}\right) \\
& +\frac{\partial}{\partial z}\left(\mu_{\mathrm{eff}} \frac{\partial u}{\partial z}\right),
\end{aligned}
$$

$y$-momentum is

$$
\begin{aligned}
P\left[\frac{\partial}{\partial x}(v u)+\frac{\partial}{\partial y}\left(v^{2}\right)+\frac{\partial}{\partial z}(v w)\right] \\
=-\frac{\partial p}{\partial y}+\frac{\partial}{\partial x}\left(\mu_{\mathrm{eff}} \frac{\partial v}{\partial x}\right)+\frac{\partial}{\partial y}\left(\mu_{\mathrm{eff}} \frac{\partial v}{\partial y}\right) \\
+\frac{\partial}{\partial z}\left(\mu_{\mathrm{eff}} \frac{\partial v}{\partial z}\right),
\end{aligned}
$$


and $z$-momentum is

$$
\begin{aligned}
P\left[\frac{\partial}{\partial x}(w u)+\frac{\partial}{\partial y}(w v)+\frac{\partial}{\partial z}\left(w^{2}\right)\right] \\
=-\frac{\partial p}{\partial z}+\frac{\partial}{\partial x}\left(\mu_{\mathrm{eff}} \frac{\partial w}{\partial x}\right)+\frac{\partial}{\partial y}\left(\mu_{\mathrm{eff}} \frac{\partial w}{\partial y}\right) \\
+\frac{\partial}{\partial z}\left(\mu_{\mathrm{eff}} \frac{\partial w}{\partial z}\right)
\end{aligned}
$$

where $u, v$, and $w$ are the velocity components in three dimensions, respectively. $\rho$ is the fluid density, and the effective viscosity, $\mu_{\text {eff }}$, is defined as $\mu_{\text {eff }}=\mu+\mu_{t}$. The turbulent viscosity depends on the selected turbulence model as well as on the specific application. In the present study, the realizable $\mathrm{k}$-e model was chosen for application here $[15,25]$.

The simulation of the two geometries was conducted using a commercial CFD software FLUENT. The design, meshing, and boundary definition of the geometries were done using the presolver software, GAMBIT. Tet/Hybrid T-grid scheme was used for the mesh generation [16]. The numbers of elements in each geometrical model were approximately 1,000,000. Grid independence test was carried out to determine the best mesh spacing for the geometrical model. The solutions are considered to be converged when all of the residuals for the continuity and momentum equations are less than or equal to $10^{-6}$.

2.2.1. Boundary Condition. The boundary condition used for the simulation is shown in Table 1.

\section{Result and Discussions}

3.1. Numerical Result. A numerical model was prepared in this study to

(1) determine the flow distribution and pressure drop at the parallel pipes and to validate the result with the data obtained from experimental setup,

(2) determine the optimum design of the tapered manifold that can give uniform water distribution through changing the diameter ratio $\left(D_{1} / D_{2}\right)$ parametrically.

CFD simulation was first performed on manifold with uniform longitudinal section having circular diameter of $10.16 \mathrm{~cm}$ (4 in) and straight flow with outlets of constant cross-sectional areas. The axial momentum would progressively decrease. This would give rise to the static pressure from the entrance to the manifold dead end. Such an increase in static pressure should favour a higher efflux through the downstream outflows. Figure 5 represents the static pressure contour for circular cross-section manifold $(D=10.16 \mathrm{~cm})$ with Reynolds number ( $\operatorname{Re}=150,000)$. It can be clearly seen from Figure 5 that the pressure along the manifold is increasing which results in nonuniformity flow.

To study the flow distribution among the parallel tubes, the dimensionless parameters, $\Phi$ and $\beta_{i}$, are used to evaluate
TABLE 1: Boundary condition for two manifolds.

\begin{tabular}{lccc}
\hline Boundary condition & Test, 1 & Test, 2 & Test, 3 \\
Reynolds number & $10 \times 10^{4}$ & $15 \times 10^{4}$ & $20 \times 10^{4}$ \\
Inlet volume rate $\mathrm{L} / \mathrm{m}$ & 500 & 750 & 1000 \\
Inlet water temperature ${ }^{\circ} \mathrm{C}$ & 20 & 20 & 20 \\
Outlet gage pressure & Zero & Zero & Zero \\
\hline
\end{tabular}

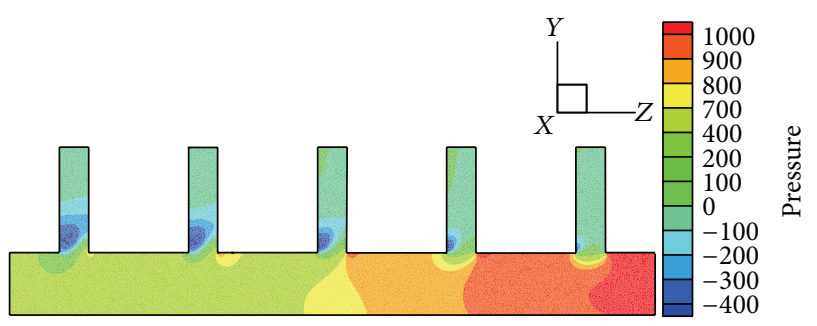

Figure 5: Pressure contour for flow in manifold with uniform longitudinal section.

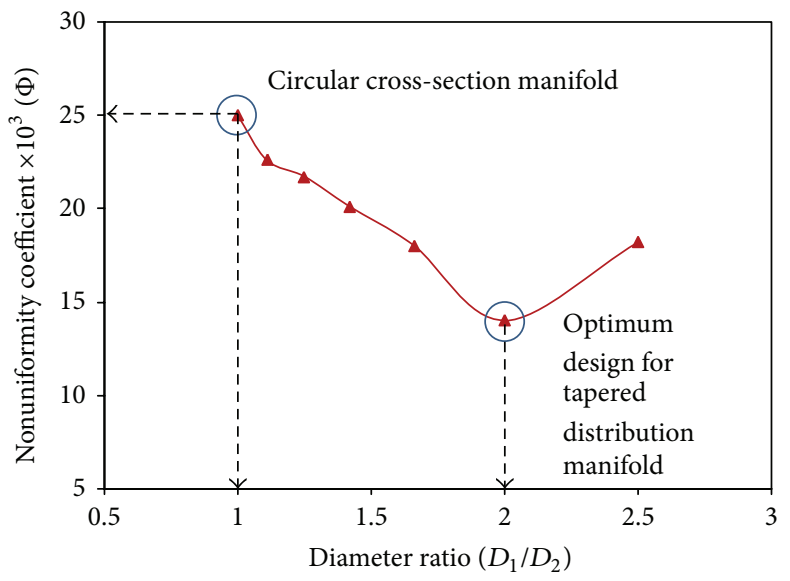

FIGURE 6: The nonuniformity coefficient $(\Phi)$ for different diameter ratio.

the flow distribution. Their definitions are given as follows [20]:

$$
\begin{gathered}
\Phi=\sqrt{\frac{\sum_{i=1}^{n}\left(\beta_{i}-\bar{\beta}\right)^{2}}{N}} \\
\bar{\beta}=\frac{Q_{i}}{Q},
\end{gathered}
$$

where $\Phi$ is the nonuniformity, $\beta_{i}$ denotes the flow ratio for $i$ th pipe, $Q_{i}$ represents volume flow rate for $i$ th pipe $\left(\mathrm{m}^{3} / \mathrm{s}\right), Q$ is total flow rate $\left(\mathrm{m}^{3} / \mathrm{s}\right), N$ is the number of parallel pipes in the manifold, and $\bar{\beta}$ is the average flow ratio for the total tubes which is defined as $\bar{\beta}=\left(\sum_{i=1}^{n} \beta_{i}\right) / N$. The large value of $\Phi$ indicates high nonuniformity. For this reason, the minimum value of nonuniformity coefficient will give the optimum configuration for the tapered manifold.

From Figure 6, the values of nonuniformities $(\Phi)$ of tapered manifold are 0.025, 0.0226, 0.0222, 0.020, 0.019, 0.014, 


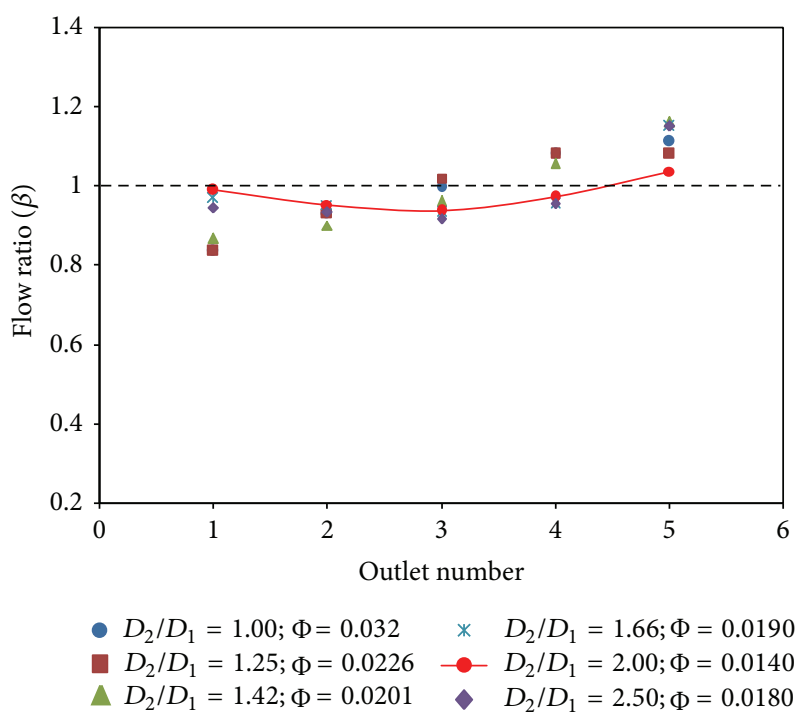

Figure 7: The flow ratio $(\beta)$ for different diameter ratio.

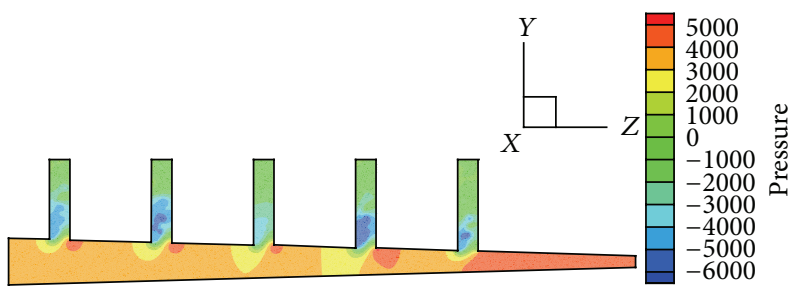

Figure 8: Pressure contour for flow in manifold with tapered longitudinal section.

and 0.0182 at diameter ratio of $1,1.1,1.25,1.42,1.66,2$, and 2.5 , respectively. The $(\Phi)$ values are lower for the manifold with circular cross-section and the corresponding value is 0.0345 as shown in Figure 6. Then the dead end diameter is reduced gradually from $10.16 \mathrm{~cm}$ ( 4 in) to $5.08 \mathrm{~cm}$ ( $2 \mathrm{in})$; the flow distribution is generally improved. The nonuniformity $(\Phi)$ decreases until it reaches a minimum value (optimal design); then it starts to increase although the diameter ratio was increased too as shown in Figure 6.

From the results shown in Figures 6 and 7, the optimum configuration of distribution manifold can be determined using diameter ratio $\left(D_{1} / D_{2}\right)$ which is equal to 2 . Figure 8 shows the pressure contour for tapered distribution manifold. The pressure along the manifold was found to be nearly uniform which resulted in a better flow distribution through to outlets.

3.2. Experimental Results. Figure 9 shows the flow distribution plots for manifold with uniform longitudinal section (diameter $10.16 \mathrm{~cm}$ ) for three values of Reynolds number $(100,000,150,000$, and 200,000). The flow through the first outlet was found to be very small compared with the last outlet as shown by pressure contours (Figure 5).

Uniform flow distribution through the manifold with tapered longitudinal section can be achieved using the design

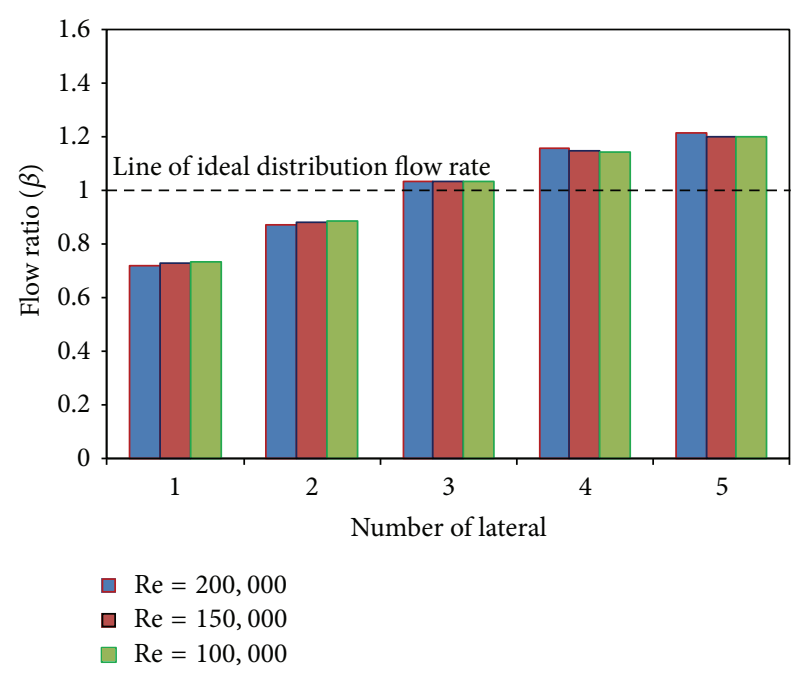

FIGURE 9: Flow distribution plot for manifold with uniform longitudinal section $(\operatorname{Re}=100,000,150,000$, and 200,000).

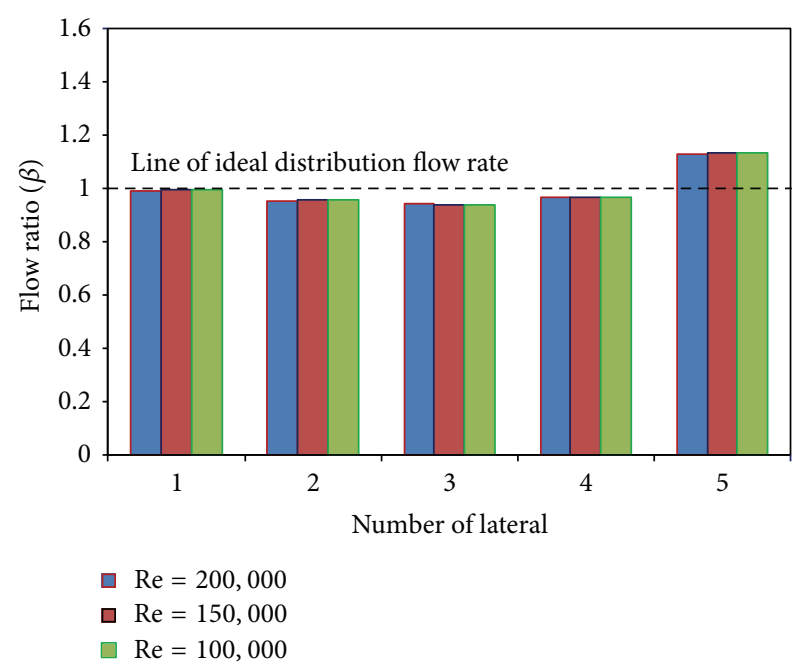

FIGURE 10: Flow distribution plot for manifold with tapered longitudinal section $(\operatorname{Re}=100,000,150,000$, and 200,000).

obtained from the numerical model. Figure 10 represents the flow distribution from manifold with tapered longitudinal section having inlet diameters of $10.16 \mathrm{~cm}$ ( 4 inch) and dead diameter of $5.08 \mathrm{~cm}$ ( 2 in). The improvement of flow distribution through the outlets is compared to that obtained from circular cross-section manifold as shown in Figures 9 and 10 .

The nonuniformity flow coefficient $(\Phi)$ was taken as a parameter to quantify the uniformity in flow distribution through the manifold outlet. The $\Phi$ can be defined using (5). Flow distribution through the outlets is better at lower $(\Phi)$ values. Table 2 shows the nonuniformity coefficient for circular and tapered manifold cross-sections from the values of Reynolds number $(100,000,150,000$, and 200,000). It can be seen that the flow distribution was severe in case 

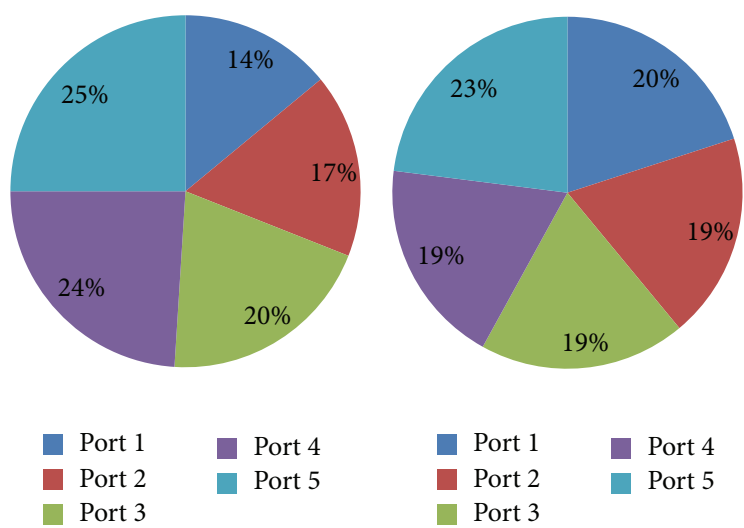

(a) Manifold with uniform longitudinal section

(b) Manifold with tapered longitudinal section

FIGURE 11: Flow rate fraction percentage for two manifold configurations ( $\operatorname{Re}=150,000, Q=750 \mathrm{~L} / \mathrm{min}$ ).

TABLE 2: The nonuniformity flow coefficient $(\Phi)$ for circular and tapered cross-section manifold.

\begin{tabular}{lcc}
\hline Manifold cross-section & $\begin{array}{c}\text { The nonuniformity flow coefficient }(\Phi) \\
\text { Circular }\end{array}$ & Tapered \\
\hline $\operatorname{Re}=100,000$ & 0.0367 & 0.0142 \\
$\operatorname{Re}=150,000$ & 0.0345 & 0.0140 \\
$\operatorname{Re}=200,000$ & 0.0340 & 0.0139 \\
\hline
\end{tabular}

of manifold of circular cross-section. The flow was evenly distributed for the manifold with tapered cross-section.

Figures 11(a) and 11(b) show the flow rate fraction of each outlet (which is the rate of outlet to the total flow rate in the manifold). For nonuniform flow, results show that the smallest flow rate occurred in outlet closest to manifold inlet while highest flow rate occurred in the last manifold outlet. Let the respective outlets be numbered as (1) which is the first outlet while the last is outlet (5). The discharge from outlet (1) is lower by $44 \%$ than outlet (5), while, for the tapered crosssection manifold, the percentage is reduced from $44 \%$ to $13 \%$.

\section{Model Validation Using Experimental Data}

Experimental tests for flow distribution from two manifolds with different configurations have been conducted. The numerical simulation results obtain by using FLUENT ${ }^{@}$ CFD package. The experimental test was conducted to measure the flow rate at the 5 outlets. The accuracy of the solution from the FLUENT ${ }^{@}$ CFD package in flow field calculation of the manifolds system is used to determine the optimal design. If the solution from the FLUENT ${ }^{@}$ CFD code cannot reproduce the actual performance of the manifold, this means that configuration for the taper distribution manifold is not optimum. The first task is thus to demonstrate the accuracy of the numerical solution. The computed and experimental flow rate distribution per outlet for $Q_{\text {total }}=750$ liter/minute $(\operatorname{Re}=1500000)$ are shown in Figures 12 and 13, respectively. It can be clearly seen that the differences in flow rates between

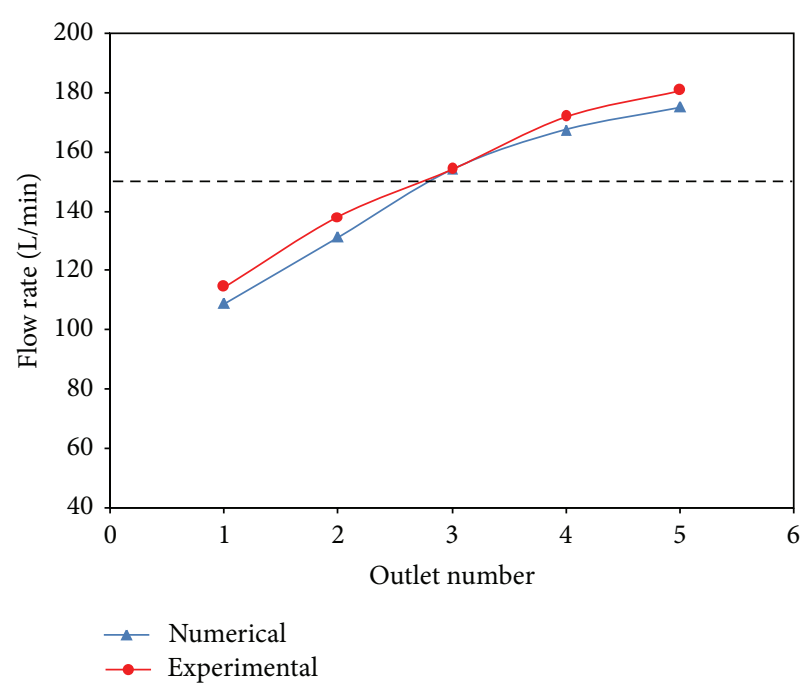

FIGURE 12: Flow distribution per outlet for manifold with uniform longitudinal section $(\mathrm{Re}=150,000, Q=750 \mathrm{~L} / \mathrm{m})$.

computed and measured are acceptable and therefore the validity of present numerical solution is evident.

\section{Conclusions}

The goal of this investigation is to evaluate the hydraulic parameter of manifold so that same rate of mass outflow can be obtained from outlet of the manifold. The CFD simulation and experimental data at different outlets and configurations, namely, circular and tapered cross-section, were carried out. Severe maldistribution was found at the outlet of the manifold with circular cross-section whereas the flow through the manifold with tapered cross-section was nearly uniform. A numerical model was used to predict the flow across each lateral for three different Reynolds numbers (i.e., 100,000, 150,000 , and 200,000) and the results were found to have the same trend compared with experimental data. The flow 


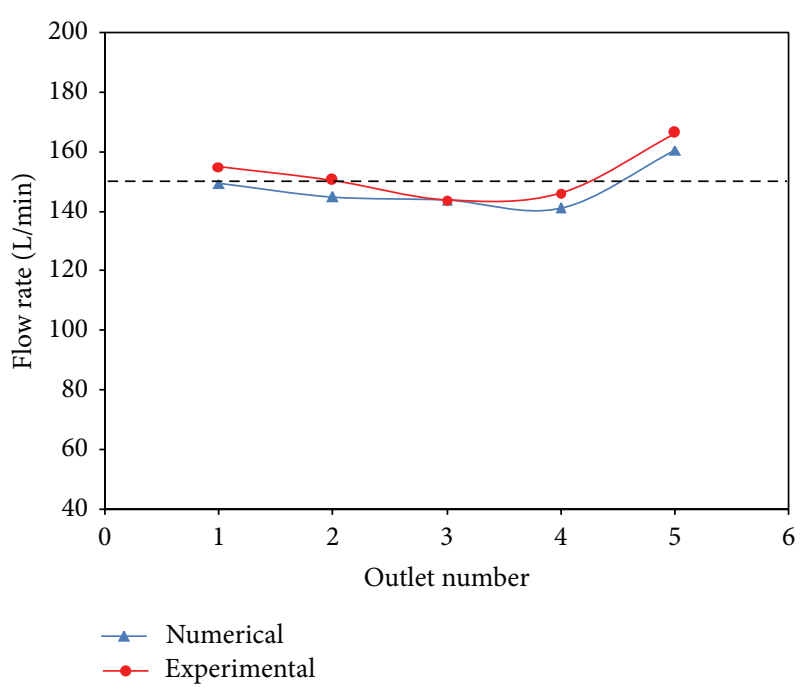

FIGURE 13: Flow distribution per outlet for manifold with tapered longitudinal section $(\operatorname{Re}=150,000, Q=750 \mathrm{~L} / \mathrm{min})$.

distribution in manifolds is independent of Reynolds number because Reynolds number was found to have slight effect on the uniformity of the mass effusion from the outlets.

\section{Conflict of Interests}

The authors declare that there is no conflict of interests regarding the publication of this paper.

\section{References}

[1] M. S. Gandhi, A. A. Ganguli, J. B. Joshi, and P. K. Vijayan, "CFD simulation for steam distribution in header and tube assemblies," Chemical Engineering Research and Design, vol. 90, no. 4, pp. 487-506, 2012.

[2] R. A. Bajura, "A model for flow distribution in manifolds," Journal of Engineering for Gas Turbines and Power, vol. 93, no. 1, pp. 7-12, 1971.

[3] R. A. Bajura and E. H. Jones Jr., "Flow distribution manifolds," Journal of Fluids Engineering, Transactions of the ASME, vol. 98, no. 4, pp. 654-666, 1976.

[4] A. K. Majumdar, "Mathematical modeling of flows in dividing and combining flow manifold," Applied Mathematical Modelling, vol. 4, no. 6, pp. 424-432, 1980.

[5] M. K. Bassiouny and H. Martin, "Flow distribution and pressure drop in plate heat exchangers-I U-type arrangement," Chemical Engineering Science, vol. 39, no. 4, pp. 693-700, 1984.

[6] M. K. Bassiouny and H. Martin, "Flow distribution and pressure drop in plate heat exchangers-II Z-type arrangement," Chemical Engineering Science, vol. 39, no. 4, pp. 701-704, 1984.

[7] S. H. Choi, S. Shin, and Y. I. Cho, "The effect of area ratio on the flow distribution in liquid cooling module manifolds for electronic packaging," International Communications in Heat and Mass Transfer, vol. 20, no. 2, pp. 221-234, 1993.

[8] S. H. Choi, S. Shin, and Y. I. Cho, "The effects of the Reynolds number and width ratio on the flow distribution in manifolds of liquid cooling modules for electronic packaging," International
Communications in Heat and Mass Transfer, vol. 20, no. 5, pp. 607-617, 1993.

[9] S. Kim, E. Choi, and Y. I. Cho, "The effect of header shapes on the flow distribution in a manifold for electronic packaging applications," International Communications in Heat and Mass Transfer, vol. 22, no. 3, pp. 329-341, 1995.

[10] A. Jiao, R. Zhang, and S. Jeong, "Experimental investigation of header configuration on flow maldistribution in plate-fin heat exchanger," Applied Thermal Engineering, vol. 23, no. 10, pp. 1235-1246, 2003.

[11] J. Wen, Y. Li, A. Zhou, and Y. Ma, "PIV investigations of flow patterns in the entrance configuration of plate-fin heat exchanger," Chinese Journal of Chemical Engineering, vol. 14, no. 1, pp. 15-23, 2006.

[12] J. C. K. Tong, E. M. Sparrow, and J. P. Abraham, "Geometric strategies for attainment of identical outflows through all of the exit ports of a distribution manifold in a manifold system," Applied Thermal Engineering, vol. 29, no. 17-18, pp. 3552-3560, 2009.

[13] P. Minqiang, Z. Dehuai, T. Yong, and C. Dongqing, "CFDbased study of velocity distribution among multiple parallel microchannels," Journal of Computers, vol. 4, no. 11, pp. 1133$1138,2009$.

[14] B. Mathew, T. J. John, and H. Hegab, "Effect of manifold design on flow distribution in multichanneled microfluidic devices," in Proceedings of the ASME Fluids Engineering Division Summer Conference (FEDSM '09), pp. 543-548, August 2009.

[15] A. W. Chen and E. M. Sparrow, "Effect of exit-port geometry on the performance of a flow distribution manifold," Applied Thermal Engineering, vol. 29, no. 13, pp. 2689-2692, 2009.

[16] V. V. Dharaiya, A. Radhakrishnan, and S. G. Kandlikar, "Evaluation of a tapered header configuration to reduce flow maldistribution in minichannels and microchannels," in Proceedings of the ASME 7th International Conference on Nanochannels, Microchannels, and Minichannels (ICNMM '09), June 2009.

[17] J. C. K. Tong, E. M. Sparrow, and J. P. Abraham, "Attainment of flowrate uniformity in the channels that link a distribution manifold to a collection manifold," Journal of Fluids Engineering, vol. 129, no. 9, pp. 1186-1192, 2007.

[18] C. Huang and C. Wang, "The design of uniform tube flow rates for Z-type compact parallel flow heat exchangers," International Journal of Heat and Mass Transfer, vol. 57, no. 2, pp. 608-622, 2013.

[19] D. W. Marquardt, "An algorithm for least-squares estimation of nonlinear parameters," Journal of Society for Industrial and Applied Mathematics, vol. 11, pp. 431-441, 1963.

[20] C.-C. Wang, K.-S. Yang, J.-S. Tsai, and I. Y. Chen, "Characteristics of flow distribution in compact parallel flow heat exchangers, part I: typical inlet header," Applied Thermal Engineering, vol. 31, no. 16, pp. 3226-3234, 2011.

[21] C. C. Wang, K. S. Yang, J. S. Tsai, and I. Y. Chen, "Characteristics of flow distribution in compact parallel flow heat exchangers, part II: modified inlet header," Applied Thermal Engineering, vol. 31, no. 16, pp. 3235-3242, 2011.

[22] D. Zeng, M. Pan, and Y. Tang, "Qualitative investigation on effects of manifold shape on methanol steam reforming for hydrogen production," Renewable Energy, vol. 39, no. 1, pp. 313322, 2012.

[23] J.-Y. Jang, Y.-X. Huang, and C.-H. Cheng, “The effects of geometric and operating conditions on the hydrogen production performance of a micro-methanol steam reformer," Chemical Engineering Science, vol. 65, no. 20, pp. 5495-5506, 2010. 
[24] H. Tuo and P. Hrnjak, "Effect of the header pressure drop induced flow maldistribution on the microchannel evaporator performance," International Journal of Refrigeration, vol. 36, pp. 2176-2186, 2013.

[25] N. Kim and H. Byun, "Effect of inlet configuration on upward branching of two-phase refrigerant in a parallel flow heat exchanger," International Journal of Refrigeration, vol. 36, no. 3, pp. 1062-1077, 2013. 

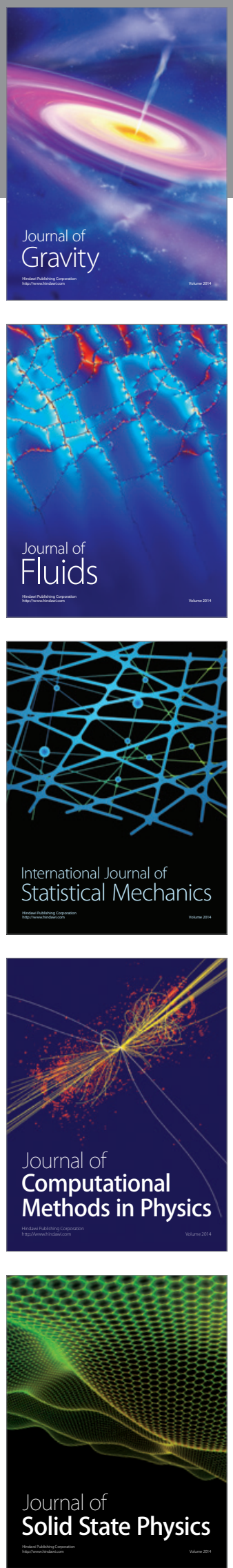

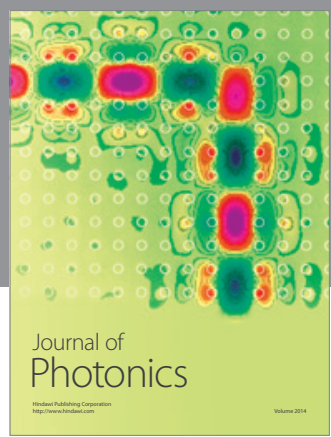

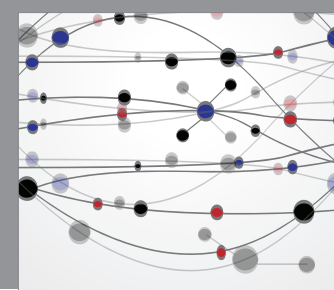

The Scientific World Journal

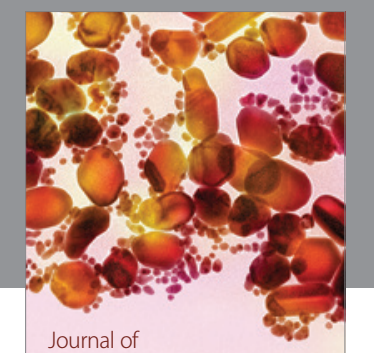

Soft Matter
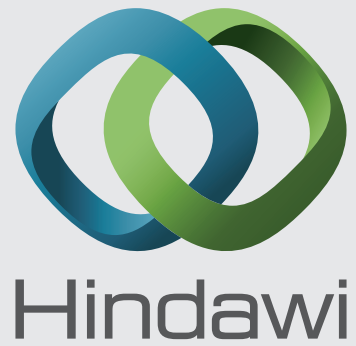

Submit your manuscripts at

http://www.hindawi.com
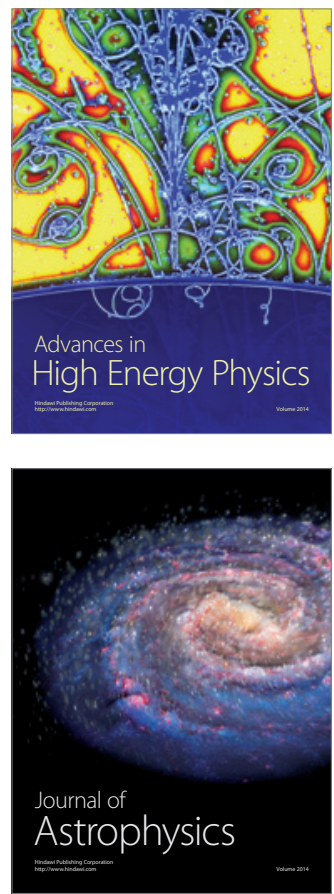
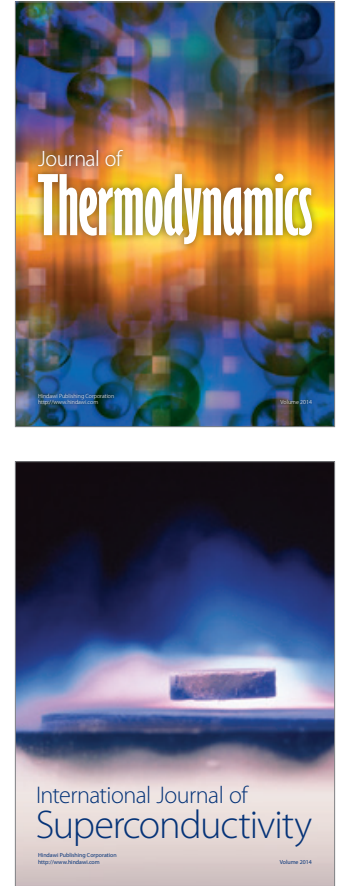
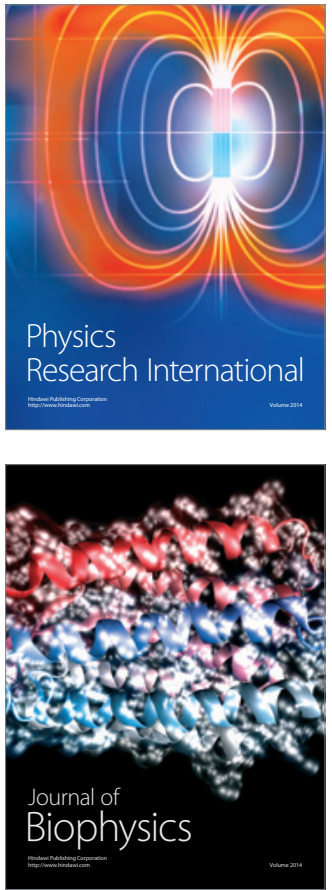
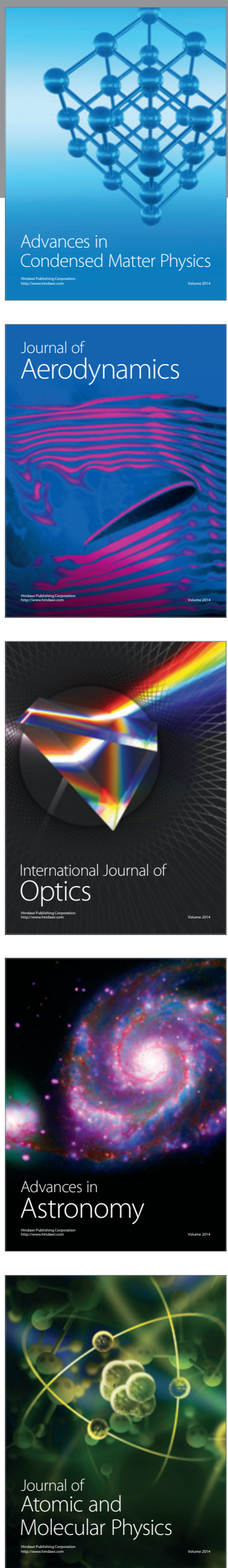